\title{
Limits of Gaudin Systems: \\ Classical and Quantum Cases
}

\author{
Alexander CHERVOV ${ }^{\dagger}$, Gregorio FALQUI ${ }^{\ddagger}$ and Leonid RYBNIKOV ${ }^{\dagger}$ \\ † Institute for Theoretical and Experimental Physics, \\ 25 Bolshaya Cheremushkinskaya Str., 117218 Moscow, Russia \\ E-mail: chervov@itep.ru,leo.rybnikov@gmail.com \\ $\ddagger$ Dipartimento di Matematica e Applicazioni, Università di Milano - Bicocca, \\ via R. Cozzi, 53, 20125 Milano, Italy \\ E-mail: gregorio.falqui@unimib.it \\ URL: http://www . matapp.unimib.it/ falqui/
}

Received November 01, 2008, in final form February 25, 2009; Published online March 09, 2009 doi:10.3842/SIGMA.2009.029

\begin{abstract}
We consider the XXX homogeneous Gaudin system with $N$ sites, both in classical and the quantum case. In particular we show that a suitable limiting procedure for letting the poles of its Lax matrix collide can be used to define new families of Liouville integrals (in the classical case) and new "Gaudin" algebras (in the quantum case). We will especially treat the case of total collisions, that gives rise to (a generalization of) the so called Bending flows of Kapovich and Millson. Some aspects of multi-Poisson geometry will be addressed (in the classical case). We will make use of properties of "Manin matrices" to provide explicit generators of the Gaudin Algebras in the quantum case.
\end{abstract}

Key words: Gaudin models; Hamiltonian structures; Gaudin algebras

2000 Mathematics Subject Classification: 82B23; 81R12; 17B80; 81R50

\section{Introduction}

The Gaudin model [21] was introduced by M. Gaudin as a spin model related to the Lie algebra $s l_{2}$, and later generalised to the case of an arbitrary semisimple Lie algebra $\mathfrak{g}$.

The Hamiltonian is

$$
H_{G}=\sum_{a=1}^{\operatorname{dim} \mathfrak{g}} \sum_{i \neq j} x_{a}^{(i)} x^{a(j)},
$$

where $\left\{x_{a}\right\}, a=1, \ldots, \operatorname{dim} \mathfrak{g}$, is an orthonormal basis of $\mathfrak{g}$ with respect to the Killing form (and $x^{a}$ its dual). These objects are regarded as elements of the polynomial algebra $\mathcal{S}\left(\mathfrak{g}^{*}\right)^{\otimes N}$ in the classical case, and as elements of the universal enveloping algebra $U(\mathfrak{g})^{\otimes N}$ in the quantum case, as

$$
x_{a}^{(i)}=1 \otimes \cdots \otimes \underbrace{x_{a}}_{i \text {-th factor }} \otimes 1 \cdots \otimes 1 .
$$

Gaudin himself found that the quadratic Hamiltonians

$$
H_{i}=\sum_{k \neq i} \sum_{a=1}^{\operatorname{dim} \mathfrak{g}} \frac{x_{a}^{(i)} x^{a(k)}}{z_{i}-z_{k}}
$$

^This paper is a contribution to the Proceedings of the XVIIth International Colloquium on Integrable Systems and Quantum Symmetries (June 19-22, 2008, Prague, Czech Republic). The full collection is available at http://www.emis.de/journals/SIGMA/ISQS2008.html 
provide a set of quantities that commute with $H_{G}$, for any choice of pairwise distinct points $z_{1}, \ldots, z_{N}$ in the complex plane. The corresponding classical quantities are "constants of the motion" for the Hamiltonian flow generated by $H_{G}$. For instance, in the $s l_{2}$ case, we have

$$
\begin{aligned}
& H_{G}=\sum_{i, j=1, i \neq j}^{N} h^{(i)} h^{(j)}+e^{(i)} f^{(j)}+f^{(i)} e^{(j)}, \\
& H_{i}=\sum_{k=1, k \neq i}^{N} \frac{h^{(i)} h^{(k)}+e^{(i)} f^{(k)}+f^{(i)} e^{(k)}}{z_{i}-z_{k}}, \quad i=1, \ldots, N .
\end{aligned}
$$

Later it was shown (see, e.g., [23]) that - in the classical case - for a general (semisimple) Lie algebra $\mathfrak{g}$ the spectral invariants of the Lax matrix

$$
L_{G}(z)=\sum_{i, a} \frac{x_{a}^{(i)} \otimes x^{a(i)}}{z-z_{i}}
$$

encode a (basically complete) set of invariant quantities. We recall that under the name spectral invariants of $L_{G}$ we mean the quantities

$$
\operatorname{Res}_{z=z_{i}}\left(z-z_{i}\right)^{k_{\alpha}} \Phi_{\alpha}\left(L_{G}\right), \quad i=1, \ldots, N,
$$

where $\Phi_{\alpha}$ is a complete set of Ad-invariant quantities for $\mathfrak{g}$, and the exponents $k_{\alpha}$ run in an appropriate set. For instance, for $\mathfrak{g}=g l(r)$, which is the case we will basically consider, we can choose $\Phi^{\alpha}\left(L_{G}(z)\right)$ to be $\operatorname{Tr}\left(L_{G}(z)^{\alpha}\right)$ (or, equivalently, the coefficients $p_{\alpha}$ of the characteristic polynomial of $L_{G}(z)$ ), with $\alpha=1,2, \ldots, r$; in this case, $k_{\alpha}$ will run from 0 to $\alpha-1$.

The meaning of "basically" complete refers to the fact that, e.g., the residues of the trace of $L_{G}^{2}(z)$ at the different points $z_{i}, i=1, \ldots, N$ are not independent, since $L_{G}(z)$ is regular at infinity. Actually, the spectral invariants of $L_{G}(z)$ generate the Poisson-commutative subalgebra of maximal possible transcendence degree in the algebra of diagonal invariants $S\left(\mathfrak{g}^{*} \oplus \cdots \oplus \mathfrak{g}^{*}\right)^{\mathfrak{g}}$. To obtain a maximal Poisson-commutative subalgebra in $S\left(\mathfrak{g}^{*} \oplus \cdots \oplus \mathfrak{g}^{*}\right)$, one has to add $\frac{1}{2}\left(\operatorname{dim} \mathfrak{g l}_{n}+\operatorname{rank} \mathfrak{g l}_{n}\right)$ independent Hamiltonians (the Mischenko-Fomenko generators for the diagonal $\mathfrak{g}$ ). This is due to the fact that, being the models we are considering homogeneous, they are invariant with respect to the diagonal adjoint action of the group $G$. We will later refer to this fact as the global $G$ invariance of the models. The enlarged set herewith defined gives to the (classical) Gaudin system the structure of a Liouville integrable system. For instance, in the case $\mathfrak{g}=s l_{2}$, one has to complement the residues of the trace of $L_{G}^{2}$ at its singular points $z_{i}$ with a further quantity, e.g. a component of the total "spin", say $S_{z}=\sum_{i=1}^{N} h^{(i)}$. It should however be noticed that the number of integrals to be added to the spectral invariants equals, independently of the number $N$ of "Gaudin generalized magnets", the rank of $\mathfrak{g}$. So, with a slight abuse of language, we will sometimes speak of a complete set of integrals referring only to the spectral invariants.

The quantum case was the subject of intense investigations. It is outside of the size of this paper to historically frame such a research line. For our purposes, it is of paramount relevance the fact that, in [18], Feigin, Frenkel and Reshetikhin proved the existence of a large commutative subalgebra $\mathcal{A}\left(z_{1}, \ldots, z_{N}\right) \subset U(\mathfrak{g})^{\otimes N}$ containing the quadratic elements $H_{i}$ (see, also, $[10,17,19,20,30,31])$. For $\mathfrak{g}=s l_{2}$, the algebra $\mathcal{A}\left(z_{1}, \ldots, z_{N}\right)$ is generated by the $H_{i}$, the additional global element $S_{z}$ and the central elements of $U(\mathfrak{g})^{\otimes N}$.

In the other cases, the algebra $\mathcal{A}\left(z_{1}, \ldots, z_{N}\right)$ has also some new generators known as higher Gaudin Hamiltonians. Their explicit construction for $\mathfrak{g}=g l(r)$ was obtained by D. Talalaev [34] and further discussed in papers by A. Chervov and D. Talalaev $[7,8]$. 
In the present paper we will discuss the problem of considering - both from the classical and from the quantum point of view - what happens when the arbitrary points $z_{1}, \ldots, z_{N}$ appearing in the Lax matrix, and in the quadratic Hamiltonians $H_{i}$ glue together; in particular, we will pay special attention to the "extreme" case, when in some sense all points collide; indeed, this extreme case gives rise to (the generalisation of) a remarkable integrable system, called the "bending flows" systems introduced and studied - for the case $\mathfrak{g}=s o(3)$ - by Kapovich and Millson [24] in their work concerning moduli spaces of polygons in $\mathbb{R}^{3}$.

\section{The classical case}

In the classical case, to shorten notations, it is customary to write the Lax matrix $L_{G}(z)(1.1)$ as $L_{G}(z)=\sum_{i=1}^{N} \frac{X_{i}}{z-z_{i}}$. It satisfies the Poisson algebra

$$
\left\{L_{G}(z) \otimes 1,1 \otimes L_{G}(u)\right\}=\left[\frac{\Pi}{z-u}, L_{G}(z) \otimes 1+1 \otimes L_{G}(u)\right],
$$

where $\Pi$ is the permutation matrix $\Pi(X \otimes Y)=Y \otimes X$.

As it is well known, this linear $r$-matrix structure (together with suitable reductions) is associated with a huge number of classical integrable systems, such as Neumann type systems and the $n$-dimensional Manakov tops, finite gap reductions of the KdV equations, as well finite gap reductions of its generalisations to arbitrary simple Lie algebras known as Gel'fand-Dickey hierarchies, Hitchin's system on singular rational curves and so on and so forth (see, e.g., [29, 1] and the references quoted therein).

Also, it is well known that the $r$-matrix Poisson brackets presented above can be seen as a kind of "shorthand notation" for the following situation:

- The phase space ${ }^{1}$ of the $N$-site classical Gaudin model is $\mathfrak{g}^{* \otimes N}$.

- The "physical" Hamiltonian is a mean field spin-spin interaction,

$$
H_{G}=\frac{1}{2} \sum_{i \neq j=1}^{N} \operatorname{Tr}\left(X_{i} \cdot X_{j}\right), \quad X_{j} \in \mathfrak{g}^{*}(\simeq \mathfrak{g}) .
$$

- The Poisson brackets defined by the $r$-matrix formula are just product of Lie-Poisson brackets on $\mathfrak{g}^{* \otimes N}$.

- The definition of the (classical) Lax matrix as $L_{G}(z)=\sum_{i=1}^{N} \frac{X_{i}}{z-z_{i}}$ defines an embedding of our phase space $\mathfrak{g}^{\otimes N}$ into a Loop space $L \mathfrak{g} \simeq \mathfrak{g}((z))$.

We now turn to the discussion of limits of the above situation when some of the points $z_{1}, \ldots, z_{N}$ collide (or glue together) in a suitable sense. We shall discuss both algebraic aspects (that is, the corresponding Lax matrices) and address also some Poisson aspects.

\subsection{Lax matrices and their limits}

To consider limits of the Gaudin algebras when some of the points $z_{1}, \ldots, z_{N}$ glue together, we can proceed as follows. We keep some (say the first $k$ ) points $z_{1}, \ldots, z_{k}$ "fixed", and let the remaining $N-k$ points glue to a new point $w$, via

$$
z_{k+i}=w+s u_{i}, \quad i=1, \ldots, N-k, \quad z_{i} \neq z_{j}, \quad u_{i} \neq u_{j}, \quad s \rightarrow 0 .
$$

We can represent this procedure as in Fig. 1.

\footnotetext{
${ }^{1}$ In this paper we will always deal with metric (or reductive) Lie algebras (and, in particular, with $g l(r)$ ), so that we will tacitly henceforth identify $\mathfrak{g}$ with $\mathfrak{g}^{*}$.
} 

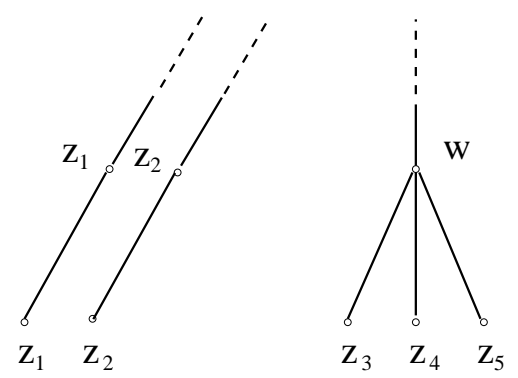

Figure 1. A gluing pattern $\pi$.

To see what happens in this limit we first observe, that obviously

$$
L_{G}(z) \rightarrow L_{2}(z)=\sum_{i=1}^{k} \frac{X_{i}}{z-z_{i}}+\frac{\sum_{i=k+1}^{N} X_{i}}{z-w}, \quad s \rightarrow 0 .
$$

Considering only this limit would however be too naïve, and indeed, we see that (even in the case of $\mathfrak{g}=g l(2))$ the number of Hamiltonians obtained from the spectral invariants of (2.2) is not sufficient to yield complete integrability.

One can notice that, in the gluing procedure, some residues of the Lax matrix should also be taken into account. That this is the case can be seen by a proper rescaling argument as follows.

Let us introduce a new variable $\tilde{z}=(z-w) / s$, so that $z=w+s \tilde{z}$, and rewrite the Lax matrix in terms of the new variable as

$$
L_{G}(z)=\sum_{i=1}^{k} \frac{X_{i}}{w+s \tilde{z}-z_{i}}+\sum_{i=k+1}^{N} \frac{X_{i}}{w+s \tilde{z}-w-s u_{i}} .
$$

Multiplying this by $s$ we can see that another Lax matrix appears for $s \rightarrow 0$, that is,

$$
s L_{G}(\tilde{z})=s\left(\sum_{i=1}^{k} \frac{X_{i}}{w+s \tilde{z}-z_{i}}+\sum_{i=k+1}^{N} \frac{X_{i}}{s\left(\tilde{z}-u_{i}\right)}\right) \rightarrow \sum_{i=k+1}^{N} \frac{X_{i}}{\tilde{z}-u_{i}}=L_{1}(\tilde{z}), \quad s \rightarrow 0 .
$$

The justification of this rescaling and limit is simple. Indeed, for $s \neq 0$, the ring of Hamiltonians obtained from $s L_{G}(\tilde{z})$ coincides with that obtained from $L_{G}(z)$. Thus the Hamiltonians obtained from the limiting Lax matrix $L_{1}(\tilde{z})$ are limits of the corresponding Hamiltonians of the Gaudin model.

Finally we conclude that starting from the Lax matrix with generic (distinct) points $z_{1}, \ldots, z_{N}$, we can associated, to the gluing of (2.1) the following pair of "Lax matrices":

$$
L_{1}(z)=\sum_{i=k+1}^{N} \frac{X_{i}}{z-u_{i}} \quad \text { and } \quad L_{2}(z)=\sum_{i=1}^{k} \frac{X_{i}}{z-z_{i}}+\frac{\sum_{i=k+1}^{N} X_{i}}{z-w} .
$$

Notice that, in the general case, we can choose the gluing procedure to be explicitly given by, e.g.,

$$
z_{k+i}=w+s\left(z_{k+i}-w\right), \quad s \in(0,1)
$$

and, using invariance w.r.t. transformation of the spectral parameter $z \rightarrow z-w$, trade the matrix $L_{1}$ of $(2.3)$ for $\tilde{L}_{1}(z)=\sum_{i=k+1}^{N} \frac{X_{i}}{z-z_{i}}$. 
In particular, in the example of the Fig. 1, we would associate, to the "generic" Lax matrix $L_{G}(z)=\sum_{i=1}^{5} \frac{X_{i}}{z-z_{i}}$ the two matrices

$$
L_{1}(z)=\frac{X_{3}}{z-z_{3}}+\frac{X_{4}}{z-z_{4}}+\frac{X_{5}}{z-z_{5}}, \quad \text { and } \quad L_{2}(z)=\frac{X_{1}}{z-z_{1}}+\frac{X_{2}}{z-z_{2}}+\frac{X_{3}+X_{4}+X_{5}}{z-w} .
$$

Proposition 1. For every choice of $w \in \mathbb{C}$ the family of spectral invariants $\mathcal{H}^{(1)}, \mathcal{H}^{(2)}$ associated with the Lax matrices $L_{1}$ and $L_{2}$ satisfy the following properties:

1. The elements of $\mathcal{H}^{(1)}, \mathcal{H}^{(2)}$ commute w.r.t. the standard (diagonal) Poisson brackets on $\mathfrak{g}^{N}$.

2. The dimension of the Poisson commutative subalgebra $\mathcal{H}_{1,2, w}$ generated by the spectral invariants $\mathcal{H}^{(1)}$ and $\mathcal{H}^{(2)}$ which are those, respectively, associated with the Lax matrices $L_{1}(z)$ and $L_{2}(z)$, coincides with that of the spectral invariants associated with the generic Lax matrix.

3. The physical Hamiltonian $H_{G}$ lies in $\mathcal{H}_{1,2, w}$.

Proof. The commutativity property (the first assertion) holds thanks to the following facts. First, it is clear that if $h_{1}, h_{2}$ are taken either both from the subfamily $\mathcal{H}^{(1)}$ or the subfamily $\mathcal{H}^{(2)}$ the assertion is trivially true. Let us suppose thus that $h_{1} \in \mathcal{H}^{(1)}$ and $h_{2} \in \mathcal{H}^{(2)}$. The fact that $\left\{h_{1}, h_{2}\right\}=0$ follows from the fact that $h_{2}$ depends on $X_{1}, X_{2}, \ldots, X_{k}$ only through the sum $\mathbf{X}_{k}=\sum_{i=1}^{k} X_{i}$, and $h_{1}$ is invariant under the diagonal action of $\mathfrak{g}$.

The second assertion holds thanks to the results of Jurčo briefly mentioned before about the number of functionally independent spectral invariants of a Lax matrix of Gaudin type, as well as to the functional dependence of $L_{2}(w)$ on the "variables" $X_{1}, \ldots, X_{k}$.

The third one can be checked by induction.

Remarks. 1) The meaning of this proposition is that, once the global $G$-invariance has been taken into account, the procedure of glueing discussed above provides new families of Liouville integrals for the "physical" Gaudin Hamiltonian $H_{G}$.

2) It is fair to say that a procedure somewhat similar to this was presented by the late V. Kuznetsov in [25]. However, possibly owing to the fact that the author restricted himself to the case of rank 1 (namely, mostly to the case $\mathfrak{g}=s o(2,1)$ ), he did not consider the need to add the further singular point $w$. This is crucial to obtain, in the general case, the correct number of Liouville integrals for the "new" system.

3) Our glueing procedure differs substantially from the one considered in [27] (see also [2]), where the limiting procedure produces higher order poles in the Lax matrix, and is associated with non-semisimple Lie-Poisson algebras, that are Inönü-Wigner contractions of the original one (see, for our case, Section 2.2).

\subsection{On the Poisson geometry of the limiting procedure}

The interplay between classical Lax matrices, Loop algebras and Poisson manifolds is nowadays well known, and was settled mainly in the works of the Leningrad's school (see, e.g. the review by Reyman and Semenov-Tyan-Shanski in [29]). It is encoded in the notion of $R$-operator as follows.

On the space $\mathfrak{g}^{*}((z))$ of Laurent polynomials with values in (the dual of) a Lie algebra $\mathfrak{g}$, there is a family of mutually compatible Poisson brackets, $\{\cdot, \cdot\}_{k}$ associated with a family of classical $R$-operators

$$
R_{k}(X(z))=\left(z^{k} X(z)\right)_{+}-\left(z^{k} X(z)\right)_{-}
$$


via the formula

$$
\{F, G\}_{k}(X)=\left\langle X,\left[R_{k}(\nabla(F)), \nabla(G)\right]\right\rangle-\left\langle X,\left[R_{k}(\nabla(G)), \nabla(F)\right]\right\rangle
$$

Spectral invariants of a Lax matrix (say, polynomially dependent on the spectral parameter $z$ ) form an Abelian Poisson subalgebra w.r.t. any of the brackets $\{\cdot, \cdot\}_{k}$.

Let us consider the space of $N$-th order polynomials $\mathfrak{g}_{N}^{*}=\mathfrak{g}^{*}[[z]] \bmod z^{N+1}$. It can be shown that (see, e.g., [29, 28])

- The brackets associated with $R_{0}, \ldots, R_{N}$ on $\mathfrak{g}^{*}((z))$ restrict to the affine subspace

$$
\mathfrak{g}_{N, A}^{*}:=\left\{X \in \mathfrak{g}^{*}((z)) \mid X(z)=z^{N+1} A+\sum_{i=0}^{N} z^{i} X_{i}\right\},
$$

where $A$ is a fixed element of $\mathfrak{g}$, and thus on $\mathfrak{g}_{N}^{*}=\mathfrak{g}_{N, \mathbf{0}}^{*}$, that is when $A=0$ which is the case we plan to consider in this paper.

- These brackets are mutually compatible and give rise to multi-Hamiltonian structures on the finite dimensional manifolds $\mathfrak{g}_{N, A}^{*}$. A straightforward observation [12] is the following: we can associate with any polynomial of degree $N \mathcal{Q}(z)=\sum_{i=0}^{N} \kappa_{i} z^{N-i}$ a Poisson bracket (of the RSTS family) via:

$$
\{\cdot, \cdot\}_{\mathcal{Q}}=\sum_{i=0}^{N} \kappa_{i}\{\cdot, \cdot\}_{i}
$$

In this setting, we can recover the standard (product) Lie-Poisson structure on $\mathfrak{g}^{\otimes N}$ as follows. Considering the "Lax map" $L_{G}=\sum_{i=1}^{N} \frac{X_{i}}{z-z_{i}}$ the diagonal structure can be obtained, in the RSTS framework, by the sum

$$
\{\cdot, \cdot\}_{\mathcal{S}}=\{\cdot, \cdot\}_{N}+\sum_{i=0}^{N-1}(-1)^{i} \sigma_{i}\{\cdot, \cdot\}_{i}
$$

the $\sigma_{i}$ being the elementary symmetric polynomials in the quantities $z_{1}, \ldots, z_{N}$. That is, the standard $r$-matrix Poisson bracket can be regarded as the one associated with the polynomial

$$
S(z)=\prod_{i=1}^{N}\left(z-z_{i}\right)=z^{N}+\sum_{i=0}^{N-1}(-1)^{i} \sigma_{i} z^{N-1} .
$$

As a side remark, one can also notice that this gives the possibility of constructing a biHamiltonian structure for the classical Gaudin case. Indeed the structure defined by the polynomial $\left(\frac{S(z)}{z}\right)_{+}$provides, together with the standard one, a bi-Hamiltonian structure for the Gaudin model, such that the spectral invariants of $L_{G}(z)$ fill in recursion relations of (generalized) Lenard-Magri type. More in detail, suitable combinations thereof give rise to anchored Lenard-Magri sequences, in the terminology of Gel'fand-Zakharevich [22].

What is more important for the present paper is that this picture suggests and gives the opportunity of studying, from this point of view, the limits of (suitable combinations of) the RSTS Poisson structures when some (and, iteratively, possibly all) poles of the Lax matrix $z_{1}, \ldots, z_{N}$ glue together. 
This means the following: if we pull back, via the map

$$
L_{G} \rightarrow L_{G, \mathrm{pol}}, \quad L_{G, \mathrm{pol}}=\prod_{i=1}^{N}\left(z-z_{i}\right)\left(\sum_{i=1}^{N} \frac{X_{i}}{z-z_{i}}\right)
$$

the RSTS Poisson brackets $\{\cdot, \cdot\}_{k}, k=0, \ldots, N$, we obtain families of linear Poisson brackets on $\mathfrak{g}_{N}^{*}=\mathfrak{g}^{*}[[z]] \bmod z^{N+1}$ that depend rationally on $\left(z_{1}, \ldots, z_{N}\right)$.

In this framework, a natural question arises, that is, whether one can associate suitable Poisson structures to the procedure of gluing (some of the) points $z_{i}$ discussed - in the Lax setting - in Section 2.1. In the example of the limit pattern depicted in Fig. 1, one of the possible Poisson structures ${ }^{2}$ that can be obtained is represented by the operator (for the sake of simplicity, we set $\left.w=z_{3}, \mathfrak{g}=g l(r)\right)$

$$
\mathcal{P}=\left[\begin{array}{ccccc}
0 & z_{23}\left[X_{1}, \cdot\right] & 0 & 0 & 0 \\
z_{23}\left[X_{1}, \cdot\right] & {\left[P_{22}, \cdot\right]} & -z_{12}\left[X_{3}, \cdot\right] & -z_{12}\left[X_{4}, \cdot\right] & -z_{12}\left[X_{5}, \cdot\right] \\
0 & -z_{12}\left[X_{3}, \cdot\right] & 0 & -\frac{z_{13} z_{34}}{z_{45}}\left[X_{3}, \cdot\right] & \frac{z_{13} z_{35}}{z_{45}}\left[X_{3}, \cdot\right] \\
0 & -z_{12}\left[X_{4}, \cdot\right] & -\frac{z_{13} z_{34}}{z_{45}}\left[X_{3}, \cdot\right] & {\left[P_{44}, \cdot\right]} & {\left[P_{45}, \cdot\right]} \\
0 & -z_{12}\left[X_{5}, \cdot\right] & \frac{z_{13} z_{35}}{z_{45}}\left[X_{3}, \cdot\right] & {\left[P_{45}, \cdot\right]} & {\left[P_{55}, \cdot\right]}
\end{array}\right]
$$

where $z_{i j}=z_{i}-z_{j}$, and

$$
\begin{aligned}
& P_{22}=z_{23}\left(X_{2}-X_{1}\right)+z_{12}\left(X_{3}+X_{4}+X_{5}\right), \\
& P_{44}=\frac{z_{13} z_{34}}{z_{45}}\left(X_{3}-\frac{z_{35}-z_{45}}{z_{45}} X_{4}-\frac{z_{13} z_{34}}{z_{45}} X_{5}\right), \\
& P_{45}=\frac{z_{13}}{z_{45}^{2}}\left(z_{35}^{2} X_{4}+z_{34}^{2} X_{5}\right), \\
& P_{55}=-\frac{z_{13} z_{35}}{z_{45}}\left(X_{3}+\frac{z_{35}}{z_{45}} X_{4}+\frac{z_{34}-z_{45}}{z_{45}} X_{5}\right) .
\end{aligned}
$$

Using the fact that both tangent vectors and one-forms on the phase space of this five-site model can be identified with five-tuples of matrices the meaning of the representation (2.4) (see also the subsequent equations (2.5), (2.6) can be illustrated as follows.

The Hamiltonian vector field associated via $\mathcal{P}$ to the one-form $\left(\alpha_{1}, \ldots, \alpha_{5}\right)$ is given by $\dot{X}_{i}=$ $\sum_{j=1}^{5}\left[P_{i j}, \alpha_{j}\right]$. E.g., $\dot{X}_{1}=z_{23}\left[X_{1}, \alpha_{2}\right]$, and so on and so forth.

It is not difficult to check that the spectral invariants of the matrices

$$
L_{1}=\frac{X_{3}}{z-z_{3}}+\frac{X_{4}}{z-z_{4}}+\frac{X_{5}}{z-z_{4}}, \quad \text { and } \quad L_{2}=\frac{X_{1}}{z-z_{1}}+\frac{X_{2}}{z-z_{2}}+\frac{X_{1}+X_{2}+X_{3}}{z-z_{3}} .
$$

do commute with respect to the Poisson brackets $\mathcal{P}$ defined by (2.4) (besides, as proven in Proposition 1, commuting w.r.t. the standard Poisson brackets).

Conjecture. For every limit pattern $\pi$ described in Section 2.1 there are suitable linear combinations $\{\cdot, \cdot\}_{\pi}$ of the Poisson brackets above that remain regular. Endowing the phase space $\mathfrak{g}^{* \otimes N}$ with these limiting brackets, one obtains bi-Hamitonian (or, possibly, multi-Hamiltonian) manifolds. The integrable systems defined, (according to the Gel'fand-Zakharevich scheme) by these

\footnotetext{
${ }^{2}$ There is two-parameter family of such structures.
} 
multi-Hamiltonian structures admit Lax representations, whose Lax matrices are exactly those constructed in Section 2.1. In other words, the spectral invariants of the Lax matrices associated with the limiting pattern $\pi$ patterns provide, w.r.t. the Poisson pencil formed out of the diagonal bracket and the bracket $\{\cdot, \cdot\}_{\pi}$ Lenard-Magri sequences (possibly in a generalised sense). Also, the brackets $\{\cdot, \cdot\}_{\pi}$ are semisimple Lie-Poisson brackets.

This conjecture has not been fully proven yet, but we have checked it in a significant number of examples. We notice that, in the "extreme gluing case", that is, when all $z_{i}$ glue according to the pattern $z_{2} \rightarrow z_{1}, z_{3} \rightarrow z_{1}\left(=z_{2}\right), z_{4} \rightarrow z_{1}\left(=z_{2}=z_{3}\right)$, .. we can obtain the following "limit" bracket which is independent of any parameter:

$$
\begin{aligned}
& \{F, G\}_{\text {limit }}=\sum_{i, j, k} r_{i j k} \operatorname{Tr}\left(\nabla F_{i}\left[X_{k}, \nabla G_{j}\right]\right) \quad \text { with } \\
& r_{i j k}=(k-1) \delta_{i j} \delta_{j k}-\theta_{(i-k)} \delta_{i j}+\theta_{(j-i)} \delta_{i k}+\theta_{(i-j)} \delta_{j k},
\end{aligned}
$$

where $\nabla F_{i},\left(\right.$ resp. $\left.\nabla G_{i}\right)$ represents the differential of $F$ (resp. $G$ ) w.r.t. the $i$-th entry $X_{i}$.

As it has been shown in [13] these Poisson brackets are still compatible with the standard $r$-matrix ones.

To elucidate the formula above, we notice that, in the 4-site case we have the following representation - by Poisson operators - of the brackets as follows:

$$
\begin{aligned}
P_{\text {standard } r}=\left(\begin{array}{cccc}
{\left[X_{1}, \cdot\right]} & & & \\
& {\left[X_{2}, \cdot\right]} & & \\
& & {\left[X_{3}, \cdot\right]} & \\
& & {\left[X_{4}, \cdot\right]}
\end{array}\right), \\
P_{\text {limit }}=\left(\begin{array}{cccc}
0 & {\left[X_{1}, \cdot\right]} & {\left[X_{1}, \cdot\right]} & {\left[X_{1} \cdot\right]} \\
{\left[X_{1}, \cdot\right]} & {\left[X_{2}-X_{1}, \cdot\right]} & {\left[X_{2}, \cdot\right]} & {\left[X_{2}, \cdot\right]} \\
{\left[X_{1}, \cdot\right]} & {\left[X_{2}, \cdot\right]} & {\left[2 X_{3}-X_{2}-X_{1}, \cdot\right]} & {\left[X_{3}, \cdot\right]} \\
{\left[X_{1}, \cdot\right]} & {\left[X_{2}, \cdot\right]} & {\left[X_{3}, \cdot\right]} & {\left[3 X_{4}-\sum_{i=1}^{3} X_{i}, \cdot\right]}
\end{array}\right) .
\end{aligned}
$$

According to the Gel'fand-Zakharevich (or Lenard-Magri) scheme, $P_{\text {standard } r}$ and $P_{\text {limit }}$ define the $g l(r)$ (as well as $\mathfrak{g}$ ) - valued generalisation of the so called Bending flows, introduced in the case corresponding to $\mathfrak{g}=\mathfrak{g l}(2)$ by Kapovich and Millson [24], and further discussed in [16].

\subsection{The Bending system}

Bending flows are defined on the moduli space $\mathcal{M}_{\mathbf{r}}$ of $(N)$-gons with fixed sides lengths $\mathbf{r}=$ $\left(r_{1}, \ldots, r_{N}\right), r_{i}>0$. In [24] it was shown, among other properties, that:

- $\mathcal{M}_{\mathbf{r}}$ is a smooth $(2 N-6)$-dimensional open manifold, whose compactification is achieved when some of the side lengths vanish.

- $\mathcal{M}_{\mathbf{r}}$ is endowed with a natural symplectic structure, since it is a symplectic quotient of products of spheres:

$$
\left\{\left(e_{1}, e_{2}, \ldots, e_{N}\right) \in S_{r_{1}}^{2} \times S_{r_{2}}^{2} \times \cdots \times S_{r_{N}}^{2}\right\} / / S O(3)
$$

where the symbol // denotes the symplectic quotient on the null manifold of the moment map defined by the diagonal $S O(3)$ action on the spheres $S_{r_{i}}$.

On this moduli space of polygons a completely integrable Hamiltonian system can be defined, indeed called the Bending system or system of bending flows. 


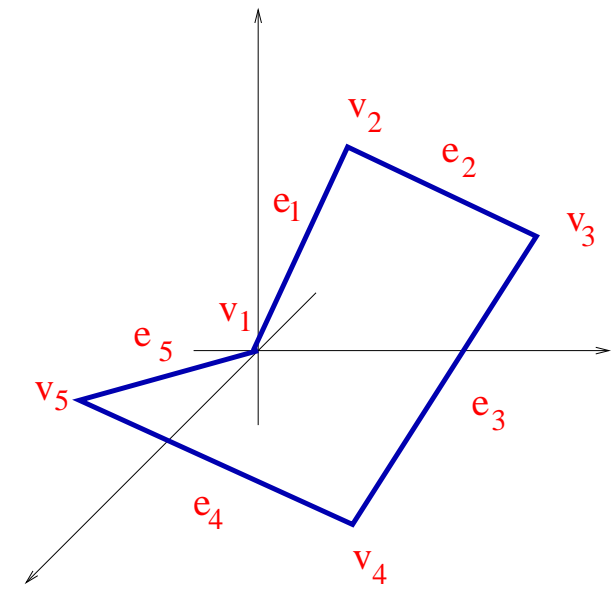

Figure 2. Polygon in $\mathbb{R}^{3}$.

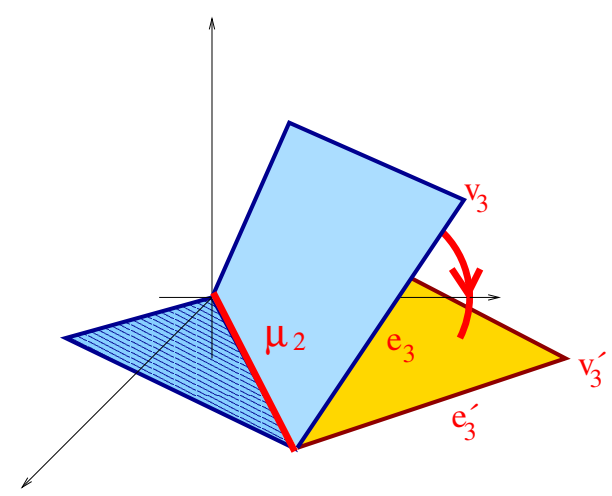

Figure 3. The Bending flows for polygons ((so(3))-case).

The action variables for such system are the lengths of diagonals stemming from one vertex, and the angle variables are (indeed) the dihedral angles (see Figs. 2 and 3). Geometrically, the flows bend one part of the polygon around the corresponding diagonal, keeping the rest fixed, whence the denomination.

In the papers $[11,13]$ the following picture was established: $\mathfrak{g}$-Bending flows can be defined on the same phase space of the $\mathfrak{g}$-Gaudin system, the link between the two (in the Kapovich Millson $N$-gon case) being the fact that $S_{r}^{2}$ is a symplectic leaf of $s o(3)^{N}$, and the condition for the polygon to close is just the choice of the special value $\mu=0$ of the image of the momentum map. Also, the following results were established:

- $\mathfrak{g}$-Bending flows admit a set of $N-1$ Lax matrices of the form

$$
L_{k}(z)=z X_{k}+\sum_{i=k+1}^{N} X_{i}, \quad k=1, \ldots, N-1 .
$$

- Hamiltonians come in "clusters", each cluster being associated with the corresponding Lax matrix. For $\mathfrak{g}=g l(r)$

$$
H_{k, m}^{a}=\underset{z=0}{\operatorname{res}} \frac{1}{z^{a+1}} \operatorname{Tr} L_{k}^{m}(z) .
$$

- Separation of variables can be performed in this scheme. Separation canonical conjugated variables come in "clusters", associated, via the bi-Hamiltonian version [15] of the Sklyanin magic recipe [33], to each of the Lax matrices. Actually, it has also been shown that integration of the Hamilton-Jacobi equation involves Abelian differentials of order independent of the number of sites. Moreover, for $\mathfrak{g}=s l(r)$ the genus of the underlying Riemann surface can be computed to be $g=\frac{r(r-1)}{2}$.

For further use, we remark that the ring of spectral invariants of a Lax matrix is, as it is well known, invariant w.r.t. rational changes in the spectral parameter and/or multiplication by a rational function of the spectral parameter. Thus, instead of the Hamiltonians (2.8) associated with the "parameter free" Lax matrices (2.7), we can consider, as an equivalent set of Hamiltonians for the generalised $g l(r)$ bending flows, the spectral invariants associated with the matrices

$$
L_{1}=\frac{X_{2}}{z-z_{2}}+\frac{X_{1}}{z-z_{1}}, \quad L_{2}=\frac{X_{3}}{z-z_{2}}+\frac{X_{1}+X_{2}}{z-z_{1}}, \quad \ldots,
$$



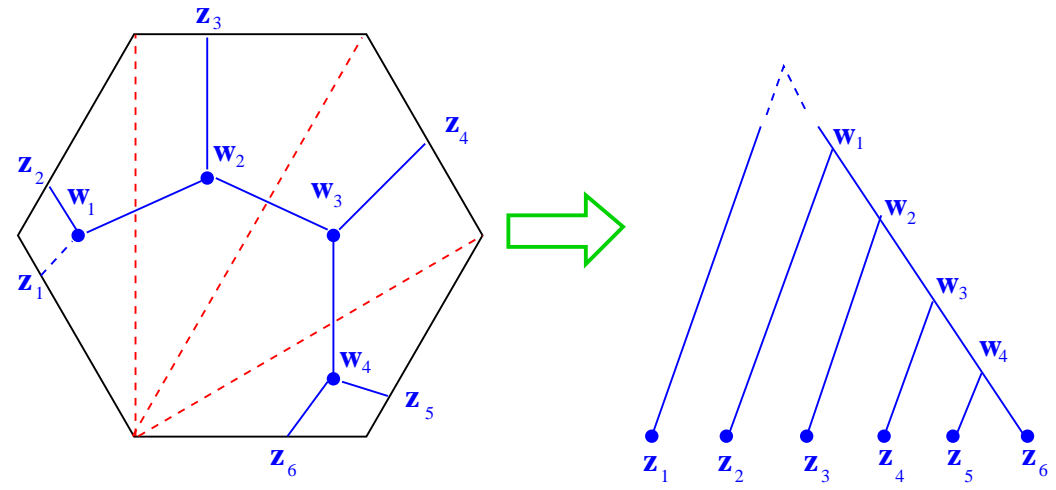

Figure 4. Triangulations and trees.

$$
L_{N-1}=\frac{X_{N}}{z-z_{2}}+\frac{\sum_{i=1}^{N-1} X_{i}}{z-z_{1}},
$$

rationally dependent on $z$. We finally want to add the following observation. As it is pictorially suggested in Fig. 4, there is a natural interpretation of our procedure in terms of triangulations of a polygon and graphs.

Actually, we have already seen (see Fig. 1) that we can interpret our procedure of glueing points in terms of building up tree-graphs out of the set of singular points of the generic Lax matrix of the Gaudin model. In particular, the glueing pattern depicted on the right of Fig. 4 corresponds to the limit in which we produce the "Bending" integrals, and, in the topological point of view, exactly gives (provided we identify the point $z_{1}$ with the root), the dual graph to the triangulation of the right hand side of the picture.

It is outside of the size of the paper to fully discuss these issues, which however the authors think being of a certain interest. It should also be noticed ${ }^{3}$ that tree diagrams corresponding to the glueing patterns described in this paper appear in the theory of moduli of pointed rational curves.

\section{The quantum case}

\subsection{FFR Gaudin algebras and their limits}

The existence of a large quantum commutative subalgebra $\mathcal{A}\left(z_{1}, \ldots, z_{N}\right) \subset U(\mathfrak{g})^{\otimes N}$ containing the Gaudin Hamiltonians $H_{i}$ was shown in [18]. As we recalled in the introduction, whenever $\mathfrak{g}$ has rank greater than one, $\mathcal{A}\left(z_{1}, \ldots, z_{N}\right)$ has new generators, besides the quadratic ones, known as higher Gaudin Hamiltonians.

Roughly speaking, the definition of $\mathcal{A}\left(z_{1}, \ldots, z_{N}\right)$ can be obtained identifying a commutative subalgebra of the enveloping algebra $U\left(\mathfrak{g} \otimes t^{-1} \mathbb{C}\left[t^{-1}\right]\right)$ as follows. To any collection $z_{1}, \ldots, z_{n}$ of pairwise distinct complex numbers, one can naturally assign the evaluation map $U\left(\mathfrak{g} \otimes t^{-1} \mathbb{C}\left[t^{-1}\right]\right) \rightarrow U(\mathfrak{g})^{\otimes N}$. The image of the commutative subalgebra in $U\left(t^{-1} \mathfrak{g}\left[t^{-1}\right]\right)$ under the composition of the above homomorphisms, denoted by $\mathcal{A}\left(z_{1}, \ldots, z_{n}\right)$, is called (quantum) Gaudin algebra.

It is useful to recall the following well known facts from the theory of Lie algebras. Let $\mathfrak{g}$ be a Lie algebra. The universal enveloping algebra $U(\mathfrak{g})$ bears a natural filtration by the degree with respect to the generators. The associated graded algebra is the symmetric algebra $S(\mathfrak{g})$ by the Poincaré-Birkhoff-Witt theorem. To every element $\xi$ in $U(\mathfrak{g})$ there corresponds uniquely its

\footnotetext{
${ }^{3}$ We thank G. Felder for an observation concerning this point.
} 
image $\bar{\xi}$ in $S(\mathfrak{g})$, since $S(\mathfrak{g})$ corresponds to the graded algebra associated with $U(\mathfrak{g})$. This element is customarily called, in the theory of Gaudin and Heisenberg spin systems/chains, the "classical limit" of $\xi$. Also, whenever $\mathfrak{g}$ is reductive, $S(\mathfrak{g}) \simeq S\left(\mathfrak{g}^{*}\right)$, and $U(\underbrace{\mathfrak{g} \oplus \mathfrak{g} \cdots \oplus \mathfrak{g}}_{N \text { times }})=\left(U(\mathfrak{g})^{\otimes^{N}}\right)$.

The problem of finding explicit representatives for generators of $\mathcal{A}\left(z_{1}, \ldots, z_{N}\right)$ in the case $\mathfrak{g}=g l(r)$ was concretely solved by Talalaev some ten years later after the paper by Feigin, Frenkel and Reshetikhin [18], by means of the following construction.

Theorem 1 (Talalaev, 2004). Let $L(z)$ be the Lax matrix of the gl(r)-Gaudin model, that is, let $L(z)$ satisfy the r-matrix commutation relations

$$
[L(z) \otimes 1,1 \otimes L(u)]=\left[\frac{\Pi}{z-u}, L(z) \otimes 1+1 \otimes L(u)\right] .
$$

Consider the differential operator in the variable $z$ "DET" $\left(\partial_{z}-L(z)\right)=\sum_{i=0, \ldots, r} Q H_{i}(z) \partial_{z}^{i}$; Then:

$$
\forall i, j \in 0, \ldots, r, \quad \text { and } \quad u, v \in \mathbb{C}, \quad\left[\left.Q H_{i}(z)\right|_{z=u},\left.Q H_{j}(z)\right|_{z=v}\right]=0 .
$$

The $Q H_{i}(z)$ 's generators of (a full set of) quantum mutually commuting quantities. We shall discuss the meaning of the symbol "DET" appearing in the formulation of Talalaev's theorem in Section 3.2, where we shall also comment on its proof.

For the moment, let us remark that the Gaudin algebra(s) defined by Feigin, Frenkel and Reshetikhin - and concretely identified by Talalaev's formula for $N$-spin $\mathfrak{g l}_{r}$ Gaudin systems explicitly depends, in general (that is when $N \geq 3$ ) on the points $z_{1}, \ldots, z_{N}$. In another parlance, its has moduli. However it holds (see [4] for the proof) the following

Proposition 2. $\mathcal{A}\left(z_{1}, \ldots, z_{N}\right)$ is invariant under permutations and under simultaneous rescalings $z_{i} \rightarrow \alpha z_{i}+\beta, \alpha, \beta \in \mathbb{C}$; thus the "two site" algebra $\mathcal{A}\left(z_{1}, z_{2}\right)$ is independent of $z_{1}, z_{2}$.

Let us now consider limits of the quantum Gaudin algebras when some of the points $z_{1}, \ldots, z_{N}$ glue together according to some pattern. Here, obviously enough, we will follow the same glueing procedure as in Section 2.

We recall that the basic procedure is that we keep some (say the first $k$ ) points $z_{1}, \ldots, z_{k}$ "fixed", and let the remaining $N-k$ points glue to a new point $w$, via

$$
z_{k+i}=w+s u_{i}, \quad i=1, \ldots, N-k, \quad z_{i} \neq z_{j}, \quad u_{i} \neq u_{j}, \quad s \rightarrow 0 .
$$

To describe this limit in the quantum case, it is useful to introduce the maps

$$
D_{k, N}:=\mathrm{id}^{\otimes k} \otimes \operatorname{diag}_{N-k}: U(\mathfrak{g})^{\otimes(k+1)} \hookrightarrow U(\mathfrak{g})^{\otimes N},
$$

defined by

$$
D_{k, N}\left(X_{1} \otimes \cdots \otimes X_{k} \otimes X_{k+1}\right)=X_{1} \otimes \cdots X_{k} \otimes \underbrace{X_{k+1} \otimes \cdots \otimes X_{k+1}}_{N-k \text { times }}
$$

and

$$
I_{k, N}:=1^{\otimes k} \otimes \mathrm{id}^{\otimes(N-k)}: U(\mathfrak{g})^{\otimes(N-k)} \hookrightarrow U(\mathfrak{g})^{\otimes N},
$$

defined by

$$
I_{k, N}\left(X_{1} \otimes \cdots X_{N-k}\right)=\underbrace{1 \otimes \cdots \otimes 1}_{k \text { times }} \otimes X_{1} \otimes \cdots \otimes X_{N-k} .
$$

Furthermore, let us define the algebra

$$
\mathcal{A}_{\left(z_{1}, \ldots, z_{k}, w\right),\left(u_{1}, \ldots, u_{N-k}\right)}:=D_{k, N}\left(\mathcal{A}\left(z_{1}, \ldots, z_{k}, w\right)\right) \cdot I_{k, N}\left(\mathcal{A}\left(u_{1}, \ldots, u_{N-k}\right)\right)
$$

In [4] it is proven the following 
Theorem 2. The algebra $\mathcal{A}_{\left(z_{1}, \ldots, z_{k}, w\right),\left(u_{1}, \ldots, u_{N-k}\right)}$ is commutative;

$$
\lim _{s \rightarrow 0} \mathcal{A}\left(z_{1}, \ldots, z_{k}, w+s u_{1}, \ldots, z+s u_{N-k}\right)=\mathcal{A}_{\left(z_{1}, \ldots, z_{k}, z\right),\left(u_{1}, \ldots, u_{N-k}\right)} .
$$

Remarks. 1) In some sense we arrive at a kind of factorisation of the limit algebra by "adding" one point. Indeed, the commutative algebra $\mathcal{A}_{\left(z_{1}, \ldots, z_{k}, w\right),\left(u_{1}, \ldots, u_{N-k}\right)}$ involves the FFR algebra associated with the points $\left(z_{1}, \ldots, z_{k}, w\right)$, and a FFR algebra related with the points $u_{1}, \ldots, u_{N-k}$. Iterating this limiting procedure described above we can obtain some new commutative subalgebras in $U(\mathfrak{g})^{\otimes N}$, just like in the classical case we found Poisson commutative subalgebras of $S(\mathfrak{g})^{\otimes^{N}}$.

2) In the case $\mathfrak{g}=g l(r)$ the classical limits (in the sense specified above) of, respectively, $I_{k, N}\left(\mathcal{A}\left(u_{1}, \ldots, u_{N-k}\right)\right)$ and $D_{k, N}\left(\mathcal{A}\left(z_{1}, \ldots, z_{k}, w\right)\right)$ coincide with the ring of the spectral invariants of the matrices

$$
L_{1}=\sum_{i=k+1}^{N} \frac{X_{i}}{z-u_{i}}, \quad \text { and } \quad L_{2}(w)=\sum_{i=1}^{k} \frac{X_{1}}{z-z_{i}}+\frac{\sum_{m=k+1}^{N} X_{m}}{z-w}
$$

already discussed in Section 2 .

In particular, as in the bending flow case, we can iterate the procedure to pass from $\mathcal{A}\left(z_{1}, z_{2}\right.$, $\left.\ldots, z_{N}\right)$ to $\mathcal{A}_{\left(z_{1}, z_{2}, \ldots, z_{N-2}, w\right)\left(z_{N-1}, z_{N}\right)}$, and finally obtain the subalgebra

$$
\mathcal{A}_{\text {lim }} \equiv \mathcal{A}_{\left(z_{1}, z_{2}\right), \ldots,\left(z_{1}, z_{2}\right)} \subset U(\mathfrak{g})^{\otimes n} .
$$

In the process, we have to use translation invariance of $\mathcal{A}\left(z_{1}, \ldots, z_{M}\right)$, as well as the property that the two site Gaudin algebra $\mathcal{A}(u, v)$ is independent of $(u, v)$.

The limit algebra (3.2) is generated by

$$
D_{1, N}\left(\mathcal{A}\left(z_{1}, z_{2}\right)\right), \quad 1 \otimes D_{1, N-1}\left(\mathcal{A}\left(z_{1}, z_{2}\right)\right), \quad \ldots, \quad 1^{\otimes(N-2)} \otimes \mathcal{A}\left(z_{1}, z_{2}\right) .
$$

More explicitly:

Proposition 3. The subalgebra $\mathcal{A}_{\lim }$ is generated by elements $H_{l, k}^{(\alpha)} \in U(g l(r))^{\otimes n}$ such that their classical "limits" $\bar{H}_{l, k}^{(\alpha)}$, are given by

$$
\bar{H}_{l, k}^{(\alpha)}\left(X_{1}, \ldots, X_{n}\right):=\operatorname{Res}_{z=0} \frac{1}{z^{\alpha+1}} \operatorname{Tr}\left(X_{k}+z \sum_{i=k+1}^{n} X_{i}\right)^{l},
$$

In other words, the classical limits of the (still unspecified) "quantum Bending Hamiltonians" do coincide with the spectral invariants of the Lax matrices $L_{k}(z)=z X_{k}+\sum_{i=k+1}^{N} X_{i}, i=2, \ldots, N$, associated with the bending flows. In turn, these can be traded for the spectral invariants of their "rational" analogs

$$
\begin{aligned}
& L_{1}(z)=\frac{X_{N-1}}{z-z_{2}}+\frac{X_{N}}{z-z_{1}}, \quad L_{2}(z)=\frac{X_{N-2}}{z-z_{2}}+\frac{X_{N}+X_{N-1}}{z-z_{1}}, \quad \ldots, \\
& L_{N-1}=\frac{X_{1}}{z-z_{2}}+\frac{\sum_{i=2}^{N} X_{i}}{z-z_{1}} .
\end{aligned}
$$

In the last part of the paper we shall show how, using Talalaev's results briefly reminded before as well as some of the results of [3] we can find a suitable quantization of the traces of the powers of the Lax matrices (3.3) (as well as of, more generally, of the quantum Lax matrices $L_{1}(z), L_{2}(z)$ associated with an elementary "gluing" procedure.

To this end, we need to introduce the notion of Manin matrix. 


\subsection{Manin matrices and the quantization of traces of Lax matrices}

We consider the (differential operator valued) matrix considered in Talalaev's Theorem 1, that is, $\mathcal{M}(z)=\partial_{z}-L(z)$. The standard linear $r$-matrix commutation relations (3.1) imply that the matrix elements of $\mathcal{M}$ satisfy special commutation relations. These are the defining commutation relations of a class of matrices (with non-commutative entries) called in [3] Manin matrices. The terminology originates from a seminal paper of Yu.I. Manin on quantum groups [26].

Manin matrices are, in a suitable sense, matrices associated with linear maps between commutative rings. Their (more operative) definition can be given as follows:

Definition 1. Let $M_{i j}$ be a matrix with elements in a non commutative (unital) ring $\mathcal{R}$; we call it a (column) Manin matrix if:

- elements in the same column commute among themselves;

- commutators of the cross terms in any $2 \times 2$ submatrix are equal:

$$
\left[M_{i j}, M_{k l}\right]=\left[M_{k j}, M_{i l}\right], \quad \text { e.g. } \quad\left[M_{11}, M_{22}\right]=\left[M_{21}, M_{12}\right] \quad \text { and so on and so forth. }
$$

Manin matrices admit a natural definition of determinant. Indeed, if $M$ is any matrix, one can in principle define "a" determinant of $M$ by column expansion:

$$
\operatorname{det} M=\operatorname{det}^{\mathrm{col}} M=\sum_{\sigma \in S_{n}}(-1)^{\sigma} \prod_{i=1, \ldots, n}^{\curvearrowright} M_{\sigma(i), i}
$$

where $S_{n}$ is the group of permutations of $n$ letters, and the symbol $\curvearrowright$ means that in the product $\prod_{i=1, \ldots, n} M_{\sigma(i), i}$ one writes at first the elements from the first column, then from the second column and so on and so forth.

In the case of Manin matrices the characteristic property of total antisymmetry of the determinant is preserved:

Proposition 4. The column determinant of a Manin matrix does not depend on the order of the columns in the column expansion, i.e.,

$$
\forall p \in S_{n} \quad \operatorname{det}^{\mathrm{col}} M=\sum_{\sigma \in S_{n}}(-1)^{\sigma} \prod_{i=1, \ldots, n}^{\curvearrowright} M_{\sigma(p(i)), p(i)} .
$$

In particular, a good notion of "DET" in the formulation of Talalaev's quantum integrals of the motion is given by the column determinant of $\mathcal{M}(z)$.

Among the properties of "ordinary" linear algebra that Manin matrices satisfy, we mention the following:

- The inverse of a Manin matrix $M$, whenever it exists, is still a Manin matrix.

- The (left) Cramer formula holds:

$$
M^{\operatorname{adj}} M=\operatorname{det}^{\mathrm{col}}(M) \mathbf{1}
$$

where $M^{\text {adj }}$ is computed - via column determinants - in the same way as in the ordinary case.

- Schur's formula for the determinant of block matrices holds:

$$
\operatorname{det}^{\mathrm{col}}\left(\begin{array}{cc}
A & B \\
C & D
\end{array}\right)=\operatorname{det}^{\mathrm{col}}(A) \operatorname{det}^{\mathrm{col}}\left(D-C A^{-1} B\right)=\operatorname{det}^{\mathrm{col}}(D) \operatorname{det}^{\mathrm{col}}\left(A-B D^{-1} C\right) .
$$


- The Cayley-Hamilton theorem, $\left.\operatorname{det}^{\mathrm{col}}(t-M)\right|_{t=M}=0$, holds true.

Further properties of Manin matrices, as well as full proofs of these facts can be found in [5]. Particularly important, for our purposes, is the the fact that a suitable redefinition of the traces of the powers of the quantum Lax matrix allows us to trade coefficients of the characteristic polynomials for these "normalised traces". The key property concerns the quantum counterparts of the classical Newton identities between these two classes of Ad-invariant quantities. The coefficients $\sigma_{i}$ of the characteristic polynomial of a $n \times n$ matrix $M$ are, as it is well known, the elementary symmetric polynomials in the eigenvalues $\lambda_{i}$ of $M$; however in the ring of symmetric polynomials in the $n$ variables $\lambda_{i}$, one can consider other sets of generators and in particular the so called power sums

$$
\tau_{i}=\sum_{k=1}^{n} \lambda_{k}^{i}=\operatorname{Tr}\left(M^{i}\right), \quad i=1, \ldots, n .
$$

In the case of matrices with commuting entries, the family $\left\{\sigma_{i}\right\}, i=1, \ldots, n$ and $\left\{\tau_{i}\right\}, i=$ $1, \ldots, n$ are related by the identities (the Newton identities):

$$
(-1)^{k+1} k \sigma_{k}=\sum_{i=0, \ldots, k-1}(-1)^{i} \sigma_{i} \tau_{k-i}
$$

The same identities hold true, in the case of Manin matrices, for the corresponding quantities.

Theorem 3. Newton identities of the form (3.4) between $\tau_{i}=\operatorname{Tr} M^{k}$ and the coefficients $\sigma_{i}$ of the expansion of $\operatorname{det}^{\mathrm{col}}(t+M)$ in powers of $t$ hold.

Proof. First one can observe that the following property, whose proof is straightforward,

$$
\operatorname{Tr}(t+M)^{\text {adj }}=\partial_{t} \operatorname{det}^{\operatorname{col}}(t+M)
$$

hold. Then we have that

$$
\begin{aligned}
& \frac{1}{t} \sum_{k=0, \ldots, \infty} \operatorname{Tr}\left((-M / t)^{k}\right)=\operatorname{Tr} \frac{1}{t+M}=\operatorname{Tr}\left(\left(\operatorname{det}^{\mathrm{col}}(t+M)\right)^{-1}(t+M)^{\mathrm{adj}}\right) \\
& =\left(\operatorname{det}^{\mathrm{col}}(t+M)\right)^{-1} \operatorname{Tr}(t+M)^{\mathrm{adj}}=\left(\operatorname{det}^{\mathrm{col}}(t+M)\right)^{-1} \partial_{t} \operatorname{det}^{\mathrm{col}}(t+M) .
\end{aligned}
$$

The result is obtained substituting $-M$ for $M$ in these formulas.

The quantization of the traces of powers of the Lax matrices can be defined according to the Newton identities and the following considerations. First, a straightforward computation that makes use of the the $r$-matrix commutation relations (3.1), proves the following

Proposition 5. Let $^{4}$

$$
\hat{L}=\sum_{i=1}^{N} \frac{\hat{X}^{i}}{z-z_{i}}
$$

be the Lax matrix for the quantum Gaudin model. For any number of sites $N$, and different $z_{i}$ the matrix $\partial_{z}-\hat{L}(z)$ is a Manin matrix.

\footnotetext{
${ }^{4}$ In these and subsequent formulæ we use the symbol ^ to stress the fact that in the quantum case $\hat{X}_{i}$ are to be regarded as elements in the universal enveloping algebra of $g l(r)$.
} 
It might be mentioned that this is not a specific property for the Gaudin system herewith considered. Indeed, Manin matrices enter other topics in (quantum) integrability; for instance, $e^{-\partial_{z}} T_{g l(r) \text {-Yangian }}(z)$ is Manin, where $T_{g l(r) \text {-Yangian }}(z)$ is the Lax (or "transfer") matrix for the Yangian algebra $Y(g l(n))$, satisfying quadratic $r$-matrix commutation relations (see [3] for more details).

The "quantization" of the traces of a Lax matrix of the form (3.5) can be achieved as follows. From Talalaev's theorem we know that the coefficients $Q H(z)$ of characteristic polynomial $\operatorname{det}^{\mathrm{col}}\left(\partial_{z}-\hat{L}(z)\right)$ do commute (at the quantum level), and from the theorem about Newton identities we know that we can trade these coefficients for traces of the powers of the corresponding Manin matrix.

Basically, we simply have to remark that we should not consider the quantities $\operatorname{Tr}\left(\hat{L}(z)^{k}\right)$, which, as it has been shown in [6] do not, generally speaking, commute, but rather the traces of powers of the Manin matrix

$$
\operatorname{Tr}\left(\left(\partial_{z}-\hat{L}(z)\right)^{k}\right)=\sum_{j=0}^{k}(Q \operatorname{Tr})_{j}^{k}(z) \partial_{z}^{k-j}, \quad k=1, \ldots, r .
$$

As it is easily seen, there is a recursion relation of the form $Q \operatorname{Tr}_{j+1}^{k}(z) \simeq Q \operatorname{Tr}_{j}^{k+1}(z)$, and hence, to obtain the expected number of independent quantities, we can consider simply the coefficients $Q \operatorname{Tr}_{k}^{k}$, that is the coefficients of zeroth order of each differential "polynomial" (3.6).

In turn, one sees that these quantities are given by the traces of matrices $\hat{L}_{k}(z)^{[n]}$, that can be called "quantum powers" of $L(z)$; these powers are defined by the Faà di Bruno formula (see, e.g., [9])

$$
\hat{L}_{k}^{[0]}(z)=\mathrm{Id}, \quad \hat{L}_{k}^{[i]}(z)=\hat{L}_{k}^{[i-1]}(z) \hat{L}_{k}(z)-\frac{\partial}{\partial z}\left(\hat{L}_{k}^{[i-1]}(z)\right) .
$$

These arguments work for any Lax matrix of "Gaudin type", that is, of the form (3.5); in particular, they hold for the (rational) Lax matrices $L_{i}(z)$ (2.9) associated with the Bending flows, since the dependence on the spectral parameter is chosen in such a way to fulfil the canonical $r$-matrix commutation relations (3.1). Thus computing the traces of the quantum powers of each of the (quantum counterparts of the) matrices $L_{i}(z)$ of $(2.9)$ one gets a quantization of the Hamiltonians of the Bending flows.

\section{Concluding remarks and open problems}

In this paper we have discussed, both in the classical and in the quantum settings, some features in the theory of (homogeneous) Gaudin models, and concentrated on the Gaudin algebras of mutually commuting quantities. We have addressed, in particular, the problem of a suitable definition of a complete set of Hamiltonians in the case when the poles of the Lax matrix $L_{G}(z)$ of the model glue together. In the classical case, we have shown that a complete set of mutually commuting Liouville integrals can be obtained by a suitable analysis of the limiting procedure on $L_{G}(z)$. We have then addressed some features of the (multi)-Hamiltonian properties of the limits of these (classical) Gaudin systems, in the framework of the Hamiltonian geometry of Loop algebras. Also, we touched the problem of framing the geometry of these systems within the combinatorial approach to the geometry of moduli spaces of pointed rational curves. In these respects, the results herewith collected are somewhat sketchy, and will be developed elsewhere.

In the quantum framework, we have followed the line of [18] as well as previous results of ours $[4,14]$. We reviewed how our gluing procedure for the poles of the Lax matrix of the Gaudin model can be applied in the quantum case to yield new quantum Gaudin algebras. Also, by means of results of [3] about the theory of the so-called Manin matrices, we have shown quite 
explicitly the procedure to define, in the quantum Gaudin algebras, a set of generators that reduce in the classical case to the usual traces of powers of the Lax matrix.

Besides the full solution of the problems in the Hamiltonian and combinatorial aspects of these systems that were just addressed in this paper, we can envisage at least two domains where the study of the limits discussed in the present paper might be of some interest. The first is the realm of Heisenberg-like models, and the theory of Yangian algebras. The second is the study of non-homogeneous Gaudin models, that, besides of their own interest in the mathematical theory of integrable (quantum) systems, proved to be important in the theory of strongly correlated electron systems (see, e.g., [32]). Due to the fact that non homogeneous systems lack global $G$-invariance, however, the results of this paper cannot be applied to that case in a straightforward manner. Work in this direction is in progress.

\section{Acknowledgments}

G.F. acknowledges support from the ESF programme MISGAM, and the Marie Curie RTN ENIGMA. The work of A.C. and L.R. has been partially supported by the Russian Federal Nuclear Energy Agency. A.C. has been partially supported by the Russian President Grant MK-5056.2007.1, by the grant of Support for the Scientific Schools 3035.2008.2, by the RFBR grant 08-02-00287a, and by the ANR grant GIMP (Geometry and Integrability in Mathematics and Physics). The work of L.R. was partially supported by the RFBR grant 050100988 -a and the RFBR grants 05-01-02805-CNRSL-a and 07-01-92214-CNRSL-a. Also, L.R. gratefully acknowledges the support from the Deligne 2004 Balzan prize in mathematics. We thank the referees for useful remarks.

\section{References}

[1] Babelon O., Bernard D., Talon M., Introduction to classical integrable systems, Cambridge Monographs on Mathematical Physics, Cambridge University Press, Cambridge, 2003.

[2] Chernyakov Yu.B., Integrable systems, obtained by point fusion from rational and elliptic Gaudin systems, Theoret. and Math. Phys. 141 (2004), 1361-1380, hep-th/0311027.

[3] Chervov A., Falqui G., Manin matrices and Talalaev's formula, J. Phys. A: Math. Theor. 41 (2008), 194006, 28 pages, arXiv:0711.2236.

[4] Chervov A., Falqui G., Rybnikov L., Limits of Gaudin algebras, quantization of bending flows, JucysMurphy elements and Gelfand-Tsetlin bases, arXiv:0710.4971.

[5] Chervov A., Falqui G., Rubtsov V., Algebraic properties of Manin matrices I, Adv. Appl. Math., to appear, arXiv:0901.0235.

[6] Chervov A., Rybnikov L., Talalaev D., Rational Lax operators and their quantization, hep-th/0404106.

[7] Chervov A., Talalaev D., Universal G-oper and Gaudin eigenproblem, hep-th/0409007.

[8] Chervov A., Talalaev D., Quantum spectral curves, quantum integrable systems and the geometric Langlands correspondence, hep-th/0604128.

[9] Dickey L., Soliton equations and Hamiltonian systems, 2nd ed., Advanced Series in Mathematical Physics, Vol. 26, World Scientific Publishing Co., Inc., River Edge, NJ, 2003.

[10] Enriquez B., Rubtsov V., Hitchin systems, higher Gaudin operators and R-matrices, Math. Res. Lett. 3 (1996), 343-357, alg-geom/9503010.

[11] Falqui G., Musso F., Gaudin models and bending flows: a geometrical point of view, J. Phys. A: Math. Gen. 36 (2003), 11655-11676, nlin.SI/0306005.

[12] Falqui G., Musso F., Bi-Hamiltonian geometry and separation of variables for Gaudin models: a case study, in SPT 2002: Symmetry and Perturbation Theory (Cala Gonone), Editors S. Abenda, G. Gaeta and S. Walcher, World Sci. Publ., River Edge, NJ, 2002, 42-50, nlin.SI/0306008.

[13] Falqui G., Musso F., On separation of variables for homogeneous sl(r) Gaudin systems, Math. Phys. Anal. Geom. 9 (2006), 233-262, nlin.SI/0402026. 
[14] Falqui G., Musso F., Quantisation of bending flows, Czechoslovak J. Phys. 56 (2006), 1143-1148, nlin.SI/0610003.

[15] Falqui G., Pedroni M., Separation of variables for bi-Hamiltonian systems, Math. Phys. Anal. Geom. 6 (2003), 139-179, nlin.SI/0204029.

[16] Flaschka H., Millson J., Bending flows for sums of rank one matrices, Canad. J. Math. 57, (2005), 114-158, math.SG/0108191.

[17] Feigin B., Frenkel E., Affine Kac-Moody algebras at the critical level and Gelfand-Dikii algebras, in Infinite Analysis, Part A, B (Kyoto, 1991), Adv. Ser. Math. Phys., Vol. 16, World Sci. Publ., River Edge, NJ, 1992, $197-215$.

[18] Feigin B., Frenkel E., Reshetikhin N., Gaudin model, Bethe ansatz and critical level, Comm. Math. Phys. 166 (1994), 27-62, hep-th/9402022.

[19] Feigin B., Frenkel E., Toledano-Laredo V., Gaudin model with irregular singularities, math.QA/0612798.

[20] Frenkel E., Affine algebras, Langlands duality and Bethe ansatz, in Proceedings XIth International Congress of Mathematical Physics (Paris, 1994), Int. Press, Cambridge, MA, 1995, 606-642, q-alg/9506003.

[21] Gaudin M., La fonction d'onde de Bethe, Collection du Commissariat à l'Énergie Atomique: Série Scientifique, Masson, Paris, 1983.

[22] Gel'fand I.M., Zakharevich I., Webs, Lenard schemes, and the local geometry of bi-Hamiltonian Toda and Lax structures, Selecta Math. (N.S.) 6 (2000), 131-183, math.DG/9903080.

[23] Jurčo B., Classical Yang-Baxter equations and quantum integrable systems, J. Math. Phys. 30 (1989), $1289-1293$.

[24] Kapovich M., Millson J., The symplectic geometry of polygons in Euclidean space, J. Differential Geom. 44 (1996), 479-513.

[25] Kuznetsov V.B., Quadrics on Riemannian spaces of constant curvature. Separation of variables and a connection with the Gaudin magnet, Theoret. and Math. Phys. 91 (1992), 385-404.

[26] Manin Yu.I., Quantum groups and noncommutative geometry, Université de Montréal, Centre de Recherches Mathématiques, Montreal, QC, 1988.

[27] Musso F., Petrera M., Ragnisco O., Algebraic extensions of Gaudin models, J. Nonlinear Math. Phys. 12 (2005), suppl. 1, 482-498, nlin.SI/0410016.

[28] Pedroni M., Vanhaecke P., A Lie algebraic generalization of the Mumford system, its symmetries and its multi-Hamiltonian structure, Regul. Chaotic Dyn. 3 (1998), 132-160.

[29] Reyman A.G., Semenov-Tian-Shansky M.A., Group-theoretical methods in the theory of finite-dimensional integrable systems, in Dynamical Systems VII, Editors V.I. Arnold and S.P. Novikov, Encyclopaedia of Mathematical Sciences, Vol. 16, Berlin, Springer, 1994, 116-225.

[30] Rybnikov L.G., The shift of invariants method and the Gaudin model, Func. Anal. Appl. 40 (2006), 188-199, math.RT/0606380.

[31] Rybnikov L.G., Uniqueness of higher Gaudin Hamiltonians, math.QA/0608588.

[32] Sierra G., Integrability and conformal symmetry in BCS model, in Statistical Field Theories (Como, 2001), NATO Sci. Ser. II Math. Phys. Chem., Vol. 73, Kluwer Acad. Publ., Dordrecht, 2002, 317-328, hep-th/0111114.

[33] Sklyanin E.K., Separation of variables - new trends, in Quantum Field Theory, Integrable Models and Beyond (Kyoto, 1994), Progr. Theoret. Phys. Suppl. (1995), no. 118, 35-60, solv-int/9504001.

[34] Talalaev D., Quantization of the Gaudin system, Funct. Anal. Appl. 40 (2006), 73-77, hep-th/0404153. 